\title{
The Peano-series solution for modeling shear horizontal waves in piezoelectric plates
}

\author{
I. Ben Salah ${ }^{1}$ a, A. Njeh ${ }^{1}$ and M.H. Ben Ghozlen ${ }^{1}$ \\ ${ }^{1}$ Laboratoire de Physique des Matériaux, FSS, BP 1171, 3000 Sfax, Tunisie
}

\begin{abstract}
The shear horizontal (SH) wave devices have been widely used in electroacoustic. To improve their performance, the phase velocity dispersion and the electromechanical coupling coefficient of the Lamb wave should be calculated exactly in the design. Therefore, this work is to analyze exactly the Lamb waves polarized in the SH direction in homogeneous plate pie.zoelectric material (PZT-5H). An alternative method is proposed to solve the wave equation in such a structure without using the standard method based on the electromechanical partial waves. This method is based on an analytical solution, the matricant explicitly expressed under the Peano series expansion form. Two types of configuration have been addressed, namely the open circuited and the short circuited. Results confirm that the SH wave provides a number of attractive properties for use in sensing and signal processing applications. It has been found that the phase velocity remains nearly constant for all values of $h / \lambda$ ( $h$ is the plate thickness, $\lambda$ the acoustic wavelength). Secondly the $\mathrm{SH}_{0}$ wave mode can provide very high electromechanical coupling. Graphical representations of electrical and mechanical amounts function of depth are made, they are in agreement with the continuity rules. The developed Peano technique is in agreement with the classical approach, and can be suitable with cylindrical geometry.
\end{abstract}

\section{Introduction}

As is well known for development of acousto-electronic devices the electro-mechanical coupling coefficient $\left(\mathrm{K}^{2}\right)$ of acoustic wave has a great importance. In this connection one of the modern lines of investigation in acoustics is the search of such wave types for which the value of $\mathrm{K}^{2}$ is maximal. At present there exist the papers showing that fundamental shear-horizontal $\left(\mathrm{SH}_{0}\right)$ acoustic waves in thin (in comparison with wavelength) piezoelectric plates possess by significantly more electromechanical coupling coefficient than surface acoustic waves (SAW) in the same material [13].

This paper presents a detailed investigation of acoustic waves propagating in piezoelectric plates. The material selected for our study was Zirconium Titanate de Plomb (PZT-5H). This material was selected since it is a strong piezoelectric material that is widely used for surface acoustic wave (SAW) devices [4]. In particular, the waves of interest here are the so-called SH (shear-horizontal) acoustic waves. The $\mathrm{SH}$ wave has a single component of particle displacement that lies in the plane

\footnotetext{
a e-mail : bs_issam@yahoo.fr
} 
of the plate surface and is at right angles to the direction of wave propagation. One of the attractive properties of such a wave is that the component of particle displacement normal to the free surface is zero. The absence of a surface normal component allows the wave to propagate in contact with a liquid without coupling and the energy leaking into the surrounding fluid can be ignored. SH wave devices can therefore be used for measuring properties of liquids and in liquid-phase-based sensors $[5,6]$. In such a plate the fundamental wave mode, namely shear horizontal $\left(\mathrm{SH}_{0}\right)$ mode can propagate, while the higher order modes are cut off [7]. The data presented in this paper is essential for the proper design of various devices based on acoustic waves propagating in PZT-5H plates.

The aim of this paper is to study the propagation behaviour of such $\mathrm{SH}$ wave in homogeneous plate piezoelectric material for which the value of $\mathrm{K}^{2}$ is maximal. The resolution of the problem passes by the analytical formulation generally proceeding from the propagator matrix $\mathrm{M}$, representing the matricant solution of the governing ordinary differential system [8]. A conventional way of the numerical treatment relies on the discretization (Thomson-Haskell) method, computing $\mathrm{M}$ as the product of exponentials of the piecewise homogeneous system matrix $Q$. This method has been further improved in [9-11]. Discussion of some other numerical methods may be found in [12].

\section{Theoretical analysis}

We consider the propagation of acoustic waves in a plate of arbitrary piezoelectric material. The geometry of the problem under consideration is shown in Figure 1. Waves propagate along the $\mathrm{x}_{1}$ direction of a piezoelectric plate bounded by planes $x_{3}=0$ and $x_{3}=-h$. The plate presents material symmetries which allow to decouple the $\mathrm{SH}$ waves (Shear Horizontal) polarized along $\mathrm{x}_{2}$-axis. For illustration a piezoelectric plate PZT-5H assumed hexagonal has been adopted. Table 1 show the elastic and electric proprieties of the piezoelectric plate.

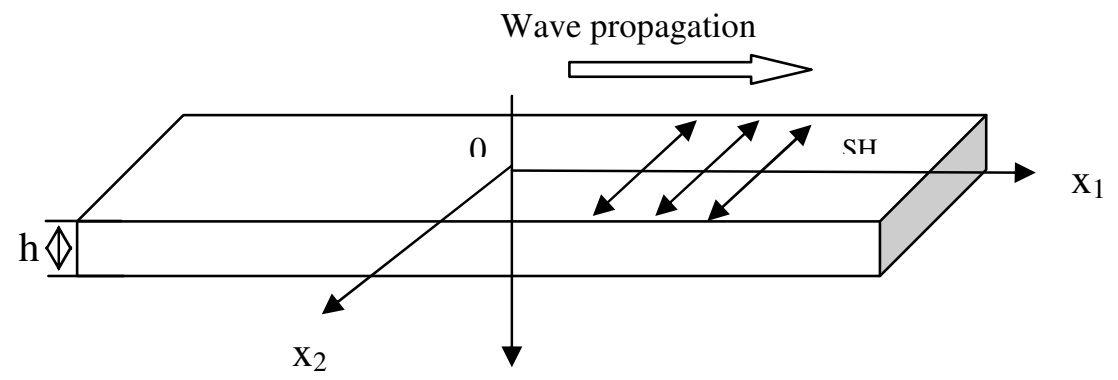

$\mathrm{X}_{3}$

Fig. 1. The geometry of the problem and coordinate system used to analyze propagation of acoustic waves in a piezoelectric plate.

Table 1. Elastic and electrical properties of piezoelectric plate (PZT-5H) [4]

\begin{tabular}{|c|c|c|c|c|}
\hline $\mathbf{C}_{\mathbf{1 1}}=\mathbf{C}_{\mathbf{2 2}}(\mathbf{G P a})$ & $\mathbf{C}_{\mathbf{1 2}}(\mathbf{G P a})$ & $\mathbf{C}_{\mathbf{1 3}}=\mathbf{C}_{\mathbf{2 3}}(\mathbf{G P a})$ & $\mathbf{C}_{\mathbf{3 3}}(\mathbf{G P a})$ & $\mathbf{C}_{\mathbf{4 4}}=\mathbf{C}_{\mathbf{5 5}}(\mathbf{G P a})$ \\
\hline 151 & 98 & 96 & 124 & 23 \\
\hline $\mathbf{e}_{\mathbf{3 1}}=\mathbf{e}_{\mathbf{3 2}}\left(\mathbf{C} / \mathbf{m}^{2}\right)$ & $\mathbf{e}_{\mathbf{3 3}}\left(\mathbf{C} / \mathbf{m}^{2}\right)$ & $\mathbf{e}_{\mathbf{1 5}}=\mathbf{e}_{\mathbf{2 4}}\left(\mathbf{C} / \mathbf{m}^{2}\right)$ & $\boldsymbol{\varepsilon}_{\mathbf{1 1}}(\mathbf{C} / \mathbf{V m})$ & $\boldsymbol{\varepsilon}_{\mathbf{3 3}}(\mathbf{C} / \mathbf{V m})$ \\
\hline-5.1 & & 17 & $15 \times 10^{-9}$ & $13.27 \times 10^{-9}$ \\
\hline
\end{tabular}

The system of governing equations involves the equation of motion, the Maxwell equation in the quasi-static approximation and the piezoelectric constitutive equations: 


$$
\begin{array}{ll}
\rho \ddot{u}_{i}=T_{i j, j} & T_{i j}=C_{i j k l} S_{k l}+e_{k i j} E_{k} \\
D_{i, j}=0 & D_{i}=e_{j k l} S_{k l}-\varepsilon_{k j} E_{k}
\end{array}
$$

In these equations, $\rho$ density and $\mathrm{C}_{\mathrm{ijkl}}$ the tensors of elastic, $\mathrm{e}_{\mathrm{ijk}}$ is the piezoelectric constants and $\varepsilon_{\mathrm{ik}}$ is the dielectric constants.

To describe the SH waves in homogeneous plate piezoelectric material, the following boundary and continuous conditions should be satisfied. It should be pointed out that two kinds of electrical boundary conditions, electrically open and shorted conditions, would be taken into account in this study. It must be reminded that the generalized stress vector $T$ includes mechanical stress vector $t_{i 3}$ $(\mathrm{i}=2)$ and the normal electrical displacement $\mathrm{D}_{3}$, in the same way the generalized displacement vector $U$ includes the mechanical displacement $u_{i}(i=2)$ and the electrical potential $\phi$. Both surfaces of the plate $\mathrm{x}_{3}=0$ and $\mathrm{x}_{3}=-\mathrm{h}$

$$
\begin{aligned}
& \mathrm{t}_{23}=0 \text { at } \mathrm{x}_{3}=0 \text { and } \mathrm{x}_{3}=-\mathrm{h} \\
& \text { Electric short case: } \phi=0 \\
& \text { Electric open case: } \sigma=0
\end{aligned}
$$

$\sigma$ refers to the charge density, it can be deduced from $\mathrm{D}_{3}$ values in the neighborhood of the both surfaces

$$
\sigma=D_{3}\left(-h^{+}\right)-D_{3}\left(-h^{-}\right)
$$

According writing of the constitutive equations and the motion equation (1), the wave equation becomes a matrix system expressed using the Thomson-Haskell parameterization of the Stroh formalism [13], with $\eta\left(\mathrm{x}_{3}\right)=[\mathrm{U}, \mathrm{T}]^{\mathrm{T}} ; \mathrm{U}=[\mathrm{i} \omega \mathrm{u}, \mathrm{i} \omega \phi]^{\mathrm{T}}$ and $\mathrm{T}=\left[\mathrm{t}_{\mathrm{i} 3}, \mathrm{D}_{3}\right]^{\mathrm{T}}$ :

$$
\frac{d}{d x_{3}} \eta\left(x_{3}\right)=i \omega Q\left(x_{3}\right) \eta\left(x_{3}\right),
$$

where the 4-component state vector $\eta\left(x_{3}\right)$ comprises the amplitudes of (6).

The general solution for the state vector can be written as:

$$
\eta\left(x_{1}, x_{3}, t\right)=\eta\left(x_{3}\right) \exp \left[i\left(k_{1} x_{1}-\omega t\right)\right]
$$

Where $\omega$ is the angular frequency, $k_{1}$ is the projection on $x_{1}$ axis of the wave vector and $\omega=2 \pi f$ is the frequency.

The explicit solution reveals the Peano expansion of the matricant. The wave equation thus formulated has an analytical solution expressed between a reference point $\left(\mathrm{x}_{1} ; 0 ; \mathrm{x}_{3}{ }_{3}\right)$ and some point of the plate $\left(\mathrm{x}_{1} ; 0 ; \mathrm{x}_{3}\right)$ in the propagation plane. This solution is called the matricant and is explicitly written under the form of the Peano series expansion [14 - 16]:

$$
M\left(x_{3}, x_{3}^{0}\right)=I+(i \omega) \int_{x_{3}^{0}}^{x_{3}} Q(\xi) d \xi+(i \omega)^{2} \int_{x_{3}^{0}}^{x_{3}} Q(\xi)\left(\int_{x_{3}^{0}}^{\xi} Q\left(\xi_{1}\right) d \xi_{1}\right) d \xi+\ldots
$$

where $I$ is the identity matrix of dimension (4×4). If the matrix components $Q\left(\mathrm{x}_{3}\right)$ are bounded in the study interval, these series are always convergent [17]. The components of the matrix $Q$ are continuous in $x_{3}$ and the study interval is bounded (thickness of the waveguide), consequently the hypothesis is always verified.

Boundary conditions: Using the propagator property of the matricant through the plate thickness, the state-vector (defined in (6)) at the second interface $\eta(-\mathrm{h})$ is evaluated from the state-vector at the first interface $\eta(0)$ as follows:

$$
\eta(-h)=M(-h, 0) \eta(0)
$$




$$
\left(\begin{array}{l}
U \\
T
\end{array}\right)_{-h}=\left(\begin{array}{ll}
M_{11} & M_{12} \\
M_{21} & M_{22}
\end{array}\right)\left(\begin{array}{l}
U \\
T
\end{array}\right)_{0}
$$

where $\eta(0)$ is a vector of 'initial' conditions on $U$ and $T$ at $x_{3}=0$, and $M_{11}, \ldots, M_{22}$ are the $2 \times 2$ blocks of $\mathrm{M}(-\mathrm{h}, 0)$.

The aim is to seek out the zeros of the determinant of $\mathrm{M}_{21}(2 \times 2)$, that leads to find the couples of values $(\mathrm{kx}, \omega)$ which describe the dispersion curves. To calculate the dispersion curves, we evaluate numerically the matricant $\mathrm{M}(-\mathrm{h}, 0)$ from the expression (8). This step requires us to truncate the Peano series and to numerically calculate the integrals. Thus, the error can be estimated and controlled [17]. For the calculations in the plate we retained 9 terms in the series and evaluate the integrals over 100 points using the Simpson's rule.

These choices are not optimized but ensure the convergence of the solution and the accuracy of the results in the range of values $\mathrm{h} / \lambda$ considered for a reasonable computation time.

\section{Results and discussions}

In a plate of a particular thickness $\mathrm{h}$, an infinite number of modes can propagate for each allowed type of wave. The velocity of each mode can be calculated as a continuous function of either the thickness-to wavelength ratio $\mathrm{h} / \lambda$ for two cases of polarizations open and short circuited "OC" and "SC". It can be seen that the $\mathrm{SH}_{0}$ mode has negligible velocity dispersion. The phase velocity nearly constant for all values of $\mathrm{h} / \lambda$. Figure 2 shows that there is one all-pass mode, it can propagate at any frequency, while all the other modes are high-pass modes. It can be seen from Figure 2 that the lowest-order $\mathrm{SH}_{0}$ wave is nearly dispersion less. The velocity of this mode lies within $2370 \pm 3.5 \mathrm{~m}$ $\mathrm{s}^{-1}$ for all values of $\mathrm{h} / \lambda$. The fact that the velocity remains nearly constant is a great advantage in the design of wide-bandwidth devices [18]. The electromechanical coupling coefficient of the SH mode calculated from equation $\mathrm{K}^{2}=2\left(\mathrm{~V}_{\mathrm{OC}}-\mathrm{V}_{\mathrm{SC}}\right) / \mathrm{V}_{\mathrm{OC}}$ is shown in Figure 3. We note that $\mathrm{K}^{2}$ reaches a value as high as $52.09 \%$ for $\mathrm{h} / \lambda=0.0253$. This is nearly eight times the value of $\mathrm{K}^{2}$ for SAWs in the widely used strong piezoelectric material PZT-5H. Due to its large coupling coefficient, devices based on this wave can operate efficiently over a wide bandwidth [18].

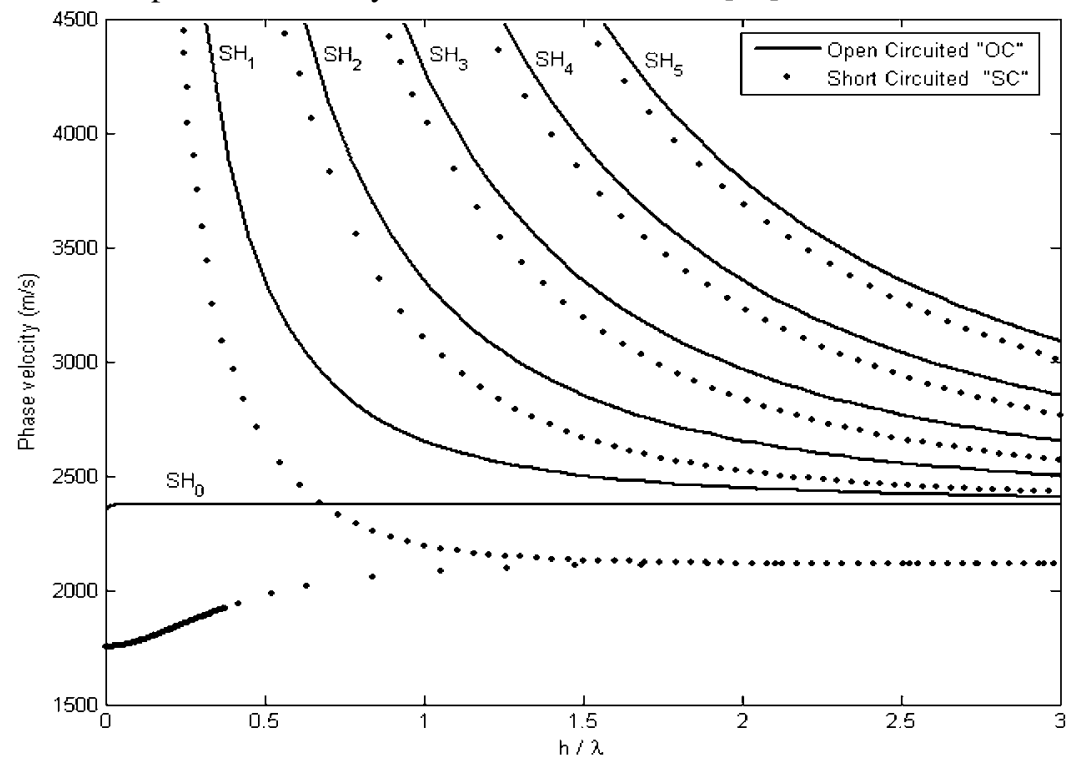

Fig. 2. Plot of phase velocity $\mathrm{V}$ versus $\mathrm{h} / \boldsymbol{\lambda}$ for the six lowest plate wave modes. There is one all-pass mode that propagate down to $h / \lambda=0$. 


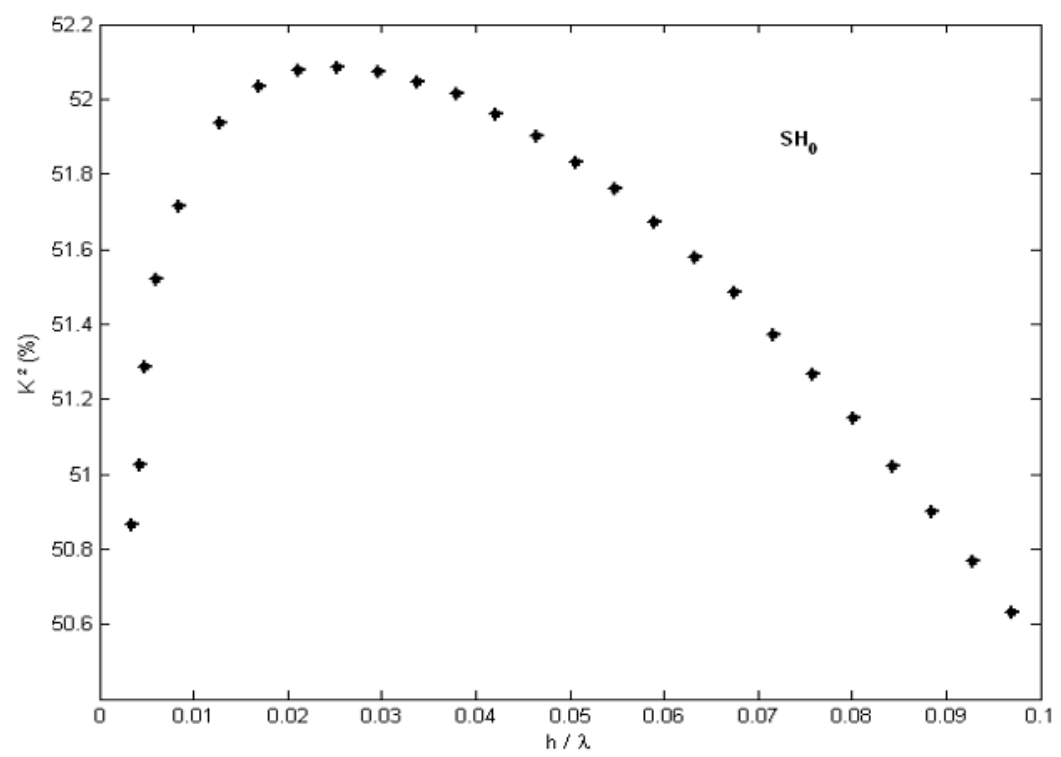

Fig. 3. Electromechanical coupling coefficient $\mathrm{K}^{2}$ as a percentage as a function of $\mathrm{h} / \mathrm{\lambda}$ for the $\mathrm{SH}_{0}$ mode.

The stress and mechanical displacement distribution in PZT-5H plate will be taken into account for fundamental mode $\mathrm{SH}_{0}$ at a frequency of $0.06 \mathrm{MHz}$ with $\mathrm{h} / \lambda=0.0253$. The variation of the mechanical displacement $\mathrm{U}_{2}$ and the stress component $\mathrm{T}_{23}$ with respect to $\mathrm{x}_{3} / \mathrm{h}$, for $\mathrm{SH}_{0}$ mode are shown in Figure 4 for electrically open. As well, the variation of the electrical displacement $\mathrm{D}_{3}$ and electrical potential $\phi$ with depth are reported in Figure 5 for electrically open. The obtained plots are in agreement with continuity rules and the free character of the lower and upper surfaces of the plate.

The representation of displacement component $\mathrm{U}_{2}$, concerning the $\mathrm{SH}_{0}$ mode, shows that the coupling is strong when the mechanical displacement parallel to the piezoelectric axis is of great amplitude for all points of the same section of the plate (Figure 4-a).

The obtained numerical results are coherent with standard methods $[9,10,17]$. The advantage of the Peano expansion method becomes essential for inhomogeneous materials and cylindrical geometry.
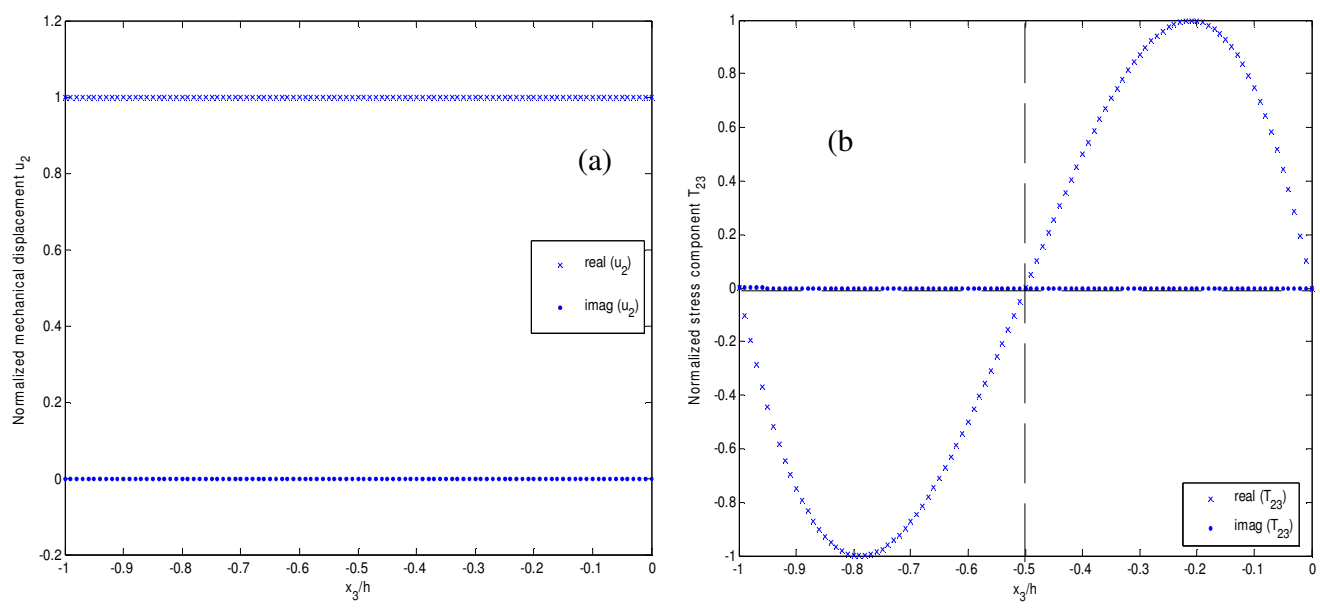

Fig. 4. Variation of normalized $\mathrm{SH}_{0}$ mechanical component $\mathrm{U}_{2}$ and $\mathrm{T}_{23}$ for different values of $\mathrm{x}_{3} / \mathrm{h}$ for both surfaces unmetallized (OC) 

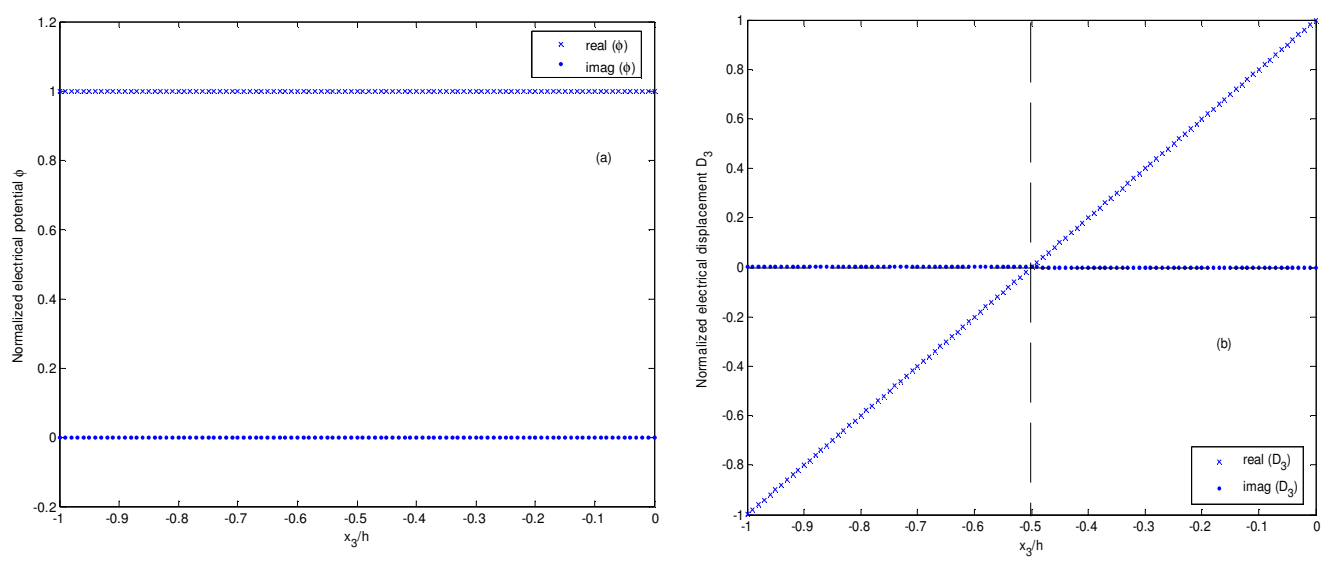

Fig. 5. Variation of normalized $\mathrm{SH}_{0}$ electrical component $\phi$ and $\mathrm{D}_{3}$ for different values of $\mathrm{x}_{3} / \mathrm{h}$ for both surfaces unmetallized (OC)

\section{Conclusion}

This paper has presented a detailed investigation of shear- horizontal acoustic waves in plates of PZT-5H. The $\mathrm{SH}$ wave can provide very high electromechanical coupling. For example, value of $\mathrm{K}^{2}$ as high as $52.09 \%$ can be obtained for $\mathrm{SH}$ waves propagating along the $\mathrm{x}_{2}$-axis of PZT-5H plate. The nearly nondispersive propagation and high value of $\mathrm{K}^{2}$ make this wave attractive for use in a variety of signal processing and sensing applications. The $\mathrm{SH}$ wave can also be advantageously employed in liquid-phase-based chemical and biological sensors [19]. In order to operate at high efficiency, SH wave devices need plates much thinner than the acoustic wavelength.

\section{References}

1. B. D. Zaitsev, S. G Joshi., I. E. Kuznetsova, IEEE Trans. Ultrason. Ferroel. and Freq. Control 46, 1298-1302 (1999).

2. I. E. Kuznetsova, B.D. Zaitsev, S.G. Joshi, I.A. Borodina, IEEE Trans. Ultrason. Ferroel. And Freq. Control. 48, $322-328$ (2001).

3. I. E. Kuznetsova, B.D. Zaitsev, S.G. Joshi, I.A. Borodina, Electronic Letters 34, N23.22802281 (1998).

4. K. Anil, D. Chakraborty, Mater. Des. 30, 1216-1222 (2009).

5. T.M. Niemczyk, S.J. Martin, G.C. Frye, A.J. Ricco, J. Appl. Phys. 64, 5002-8 (1988).

6. S.J. Martin, A.J. Ricco, T.M. Niemczyk, G.C. Frye, Sensors Actuators 20, 253-68 (1989).

7. B.D. Zaitsev, S.G. Joshi, I.E. Kuznetsova, Smart Mater. Struct. 739-744 (1997).

8. M.C. Pease III, Methods of Matrix Algebra, Academic Press, New York, (1965).

9. S.I. Rokhlin, L. Wang, J. Acoust. Soc. Am. 112, 822-834 (2002).

10. B. Hosten, M. Castaings, Ultrasonics 41, 501-507 (2003).

11. Eng Leong Tan, J. Acoust. Soc. Am. 119, 45-53 (2006).

12. G.R. Liu, Z.C. Xi, CRC Press, Boca Raton, FL, (2002).

13. A. N. Stroh, Journal of Mathematics and Physics 41, 77-103 (1962).

14. F. Gantmacher, the theory of matrices (Wiley Interscience) (1959).

15. G. Peano, Mathematische annallen 32, 450-456 (1888).

16. M. Pease, Methods of matrix algebra (Academic Press) (1965).

17. C. Baron, Thesis, Université Bordeaux 1 (2005).

18. K. Michio, A. Junya, H. Hideya, I. Mamoru, Jap. J. A. Phy., 40, 3722 (2001).

19. F. Josse, F. Bender, W. Richard, Anal. Chem., 73 (24), 5937-5944 (2001). 